\title{
PRINCIPIOS DEL CAMBIO LINGÜÍSTICO Una contribución sincrónica a la lingüística histórica ${ }^{1}$
}

\author{
Rocío CaRavedo \\ Università di Pisa
}

Las páginas siguientes se proponen presentar de modo razonado los principales problemas teónico-metodológicos surgidos de una lectura minuciosa del más reciente estudio de William Labov sobre los factores sociales que originan el cambio linguiístico, problemas que no sólo se circunscriben al ámbito particular de la teoría de la variación, también llamada sociolinguística, sino que involucran la linguística histórica, e incluso un dominio de mayor alcance como es el de la linguística general.

Si en los primeros estudios de Labov el foco perceptivo se orientaba hacia la organización de la variación intema de las lenguas, en los últimos el interés se fija en la variación inestable, considerada como un primer paso hacia la modificación de las mismas. El poner el cambio en el centro de la atención no significa en modo alguno abandonar los objetivos primarios de la sociolingüística, sino que constituye, más bien, un intento por comprender en su totalidad el proceso evolutivo de las lenguas, respecto del cual variación y cambio pueden considerarse como dos fases interconectadas.

Aquí abordaré de modo selectivo, y sin ocultar un sesgo personal, algunas cuestiones que considero particularmente relevantes entre las muchas que plan-

1 A propósito de William Labov, Principles of Linguistic Change. Vol II. Sacial Factors. Oxford, Blackwell, 2001, 572 págs. Este libro constituye el segundo volumen de un gran proyecto sobre el cambio, tema que ha estado siempre presente en las preocupaciones teoricas del autor desde sus primeros trabajos. Cf. uno de los más significativos, en coautoría con su maestro Uriel Weinreich y con su entonces compañero de estudios, Marvin Herzog, «Empirical Foundations for a Theory of Linguistic Change», en W. Lehmann y Y. Malkied (eds.), Directions for Historical Linguistics. Austin, University of Texas Press, págs. 97-195. Cf. también el primer volumen: Principles of Linguistic Change. Internal Factors. Oxford, Blackwell, 1994 (versión espanola de Pedro Martín Butragueño, Principios del cambio linguístico. Factores Intemos. Madrid, Gredos, 1996). No es, pues, meramente anecdótico señalar que ambos tomos estén precisamente dedicados al maestro.

$R F E$, LXXXIII, 2003, 1. ${ }^{\circ}-2{ }^{\circ}$, págs. 39-62 
tea una obra de la riqueza y de la envergadura de ésta. Si bien no me detendré en el análisis de los procedimientos metodológicos ni de las estrategias empíricas particulares, no dejo de apuntar que la solvencia de esta investigación se funda en la observación sistematizada de un conjunto apreciable de datos rigurosamente clasificados y cuantificados. No se encuentran, pues, intuiciones sin apoyo empírico, y toda generalización constituye un resultado de inferencias inductivas cuidadosamente explicitadas. Sin embargo, contra lo que pudiera desprenderse de este tipo de lógica, y más allá de los fenómenos circunscritos a una lengua particular como el inglés en su modalidad americana, el autor plantea problemas de largo alcance relacionados con el descubrimiento de los principios generales que regulan tanto la estabilidad cuanto la inestabilidad de las lenguas ${ }^{2}$. Por un lado, la estabilidad, en la perspectiva del autor, puede referirse a la invariación y a la variación estable, y por otro, la inestabilidad atañe a cualquier modificación que al atentar contra el orden establecido (en cualquiera de sus dos formas: invariación o variación estable) marque asimismo el inicio del cambio (change in progress).

Todo cambio presupone, pues, variación, mientras que no toda variación desemboca en cambio, pudiendo incluso permanecer estable. Sin embargo, si aceptamos esta relación unidireccional entre ambos hechos, es posible considerar la variación más bien como expresión de invariabilidad, cuando se estabiliza sin resolverse jamás en una mutación definida. Al parecer, esta dirección interpretativa acortaría la distancia existente entre la linguística de sesgo invariacionista o inmanentista (i.e. autónoma) y la variacionista, y entrecruzaría sus objetivos. La variación actuaría, pues, como una especie de bisagra, articulando dos órdenes aparentemente contradictorios, como la permanencia y la transformación. En todo caso, la preocupación central de Labov en la obra que nos ocupa conduce a un redimensionamiento de la lingüística de la variación (a mi modo de ver, impropiamente llamada «sociolingüística»), la cual, en razón de su foco de interés general, se sitúa plenamente en el ámbito de la lingüística histórica, entendida ésta como un dominio amplio en el cual el presente forma parte de la historia.

\footnotetext{
${ }^{2}$ Más precisamente, la base empírica de la que parte el autor para este estudio es el proyecto sobre el cambio lingïístico en curso en la ciudad de Filadelfia (Project on Linguistic Change and Variation), que comenzo en 1970, y que se apoya en un amplio corpus de entrevistas, a través de un observador participante, en diversos barrios de la ciudad, combinadas con encuestas telefónicas aleatorias a 112 informantes. El autor ha ido actualizando a través de los años los datos primarios y se ha valido, además, de la información proporcionada por su Atlas Lingǘrstico de Norteamérica (The Atlas of North American English: Phonology and Sound Change), en proceso de publicación. En muchos casos remite a las investigaciones en el marco de su primer proyecto sobre la variación del inglés en Nueva York, que data de los años sesenta. Para reforzar sus conjeturas utiliza también los resultados de investigaciones realizadas en diversos lugares de Norteamérica y Gran Bretaña para el inglés, acudiendo en algunos casos a comparaciones con estudios provenientes de otras lenguas, entre las cuales se encuentra el español.
} 
Desde la perspectiva laboviana la variación puede entonces representar o no un primer paso hacia el cambio, de modo que la estabilidad de las lenguas descansa en cierto equilibrio interno de la variación, y el cambio se produce sólo cuando la estabilidad se rompe en algún punto del espacio a causa del comportamiento de ciertos grupos sociales ${ }^{3}$ que se desvían de modo abrupto de las tendencias de los demás integrantes de la sociedad.

\section{El ESTAtUTO DE LA VARIABLE ESTABLE COMO UNIDAD ANALÍtica, PARADIGMÁTICA Y AISLADA}

Uno de los aspectos más debatidos de la lingüística de la variación es sin duda el concepto de unidad analítica que sirve de punto de referencia al trabajo sociolinguístico. Tal concepto - como lo he dicho ya en otro lugar- resulta de una operación de tipo paradigmático-segmentalista, que puede sostenerse en el nivel fonológico, incluso en el léxico, y en ciertos aspectos del morfologico, pero que fracasa cuando se trata de aplicar al plano sintáctico, donde no son unidades las que se ponen en juego, sino complejos combinatorios de muchos componentes de distinta jerarquía ensamblados entre $s^{4}{ }^{4}$. Obviamente la variación sintáctica escapa a los criterios de medición que se muestran adecuados a segmentos fonológicos o a ciertas unidades morfológicas de inventarios más bien cerrados y con significados de contornos nítidos.

De hecho Labov no hace el menor intento de esconder el papel privilegiado que el plano fonológico desempeña en la variación. $Y$ antes bien, en este estudio vuelve a insistir en la pertinencia decisiva de lo fonológico en la evolución de las lenguas: el cambio - según él- es más susceptible de presentarse en este plano que en el sintáctico, si bien puede acarrear consecuencias que vayan a la larga mucho más allá de él, involucrando unidades gramaticales superiores como las morfosintácticas y las léxicas. No se plantea Labov, por lo menos en este volumen, la posibilidad de evoluciones de tipo cognoscitivo que lleven a reestructuraciones de las categorías gramaticales, tales como las implicadas en los procesos de gramaticalización o desgramaticalización. Aun si el cambio fonológico no llegara a repercutir en las configuraciones morfológicas de la lengua, el autor reconoce que de alguna manera el sistema de alofonos y sus correspondientes fonemas como unidades estructurales queda también involucrado, lo cual quiere decir que no se trata de meros cambios en el orden ma-

\footnotetext{
${ }^{3}$ Hago aquí la siguiente precisión: al referirme a los 'grupos sociales' no aludo al concepto de clases sociales, sino de modo general a cualquier tipo de colectividad.

4 Cf. Rocío Caravedo, Lingatistica del corpus. Cuestiones teórico-metodológicas aplicadas al español. Universidad de Salamanca, 1999.
} 
terial, sino de cambios que alcanzan un orden estructural (cf. págs. 28-29). De hecho, son sólo fenómenos fonológicos los que ocupan la atención de Labov en los dos volúmenes de Principles y se refieren al complejo subsistema vocálico del inglés, el punto más sujeto a variación en esta lengua. Con todo, la restricción programática del estudio del cambio a lo fonológico corre el peligro de dejar de lado las diversas mutaciones de tipo sintáctico que han sufrido las lenguas (y el inglés es una de ellas) a lo largo de su evolución.

Por otro lado, en razón del tema central de este trabajo, no se toca el problema de la unidad de significado de la variable, que durante varios años fue el punto más debatido y conflictivo de los postulados variacionistas. $Y$ esto parece coherente con el hecho de que el cambio por su propia naturaleza va en contra de la preservación de las unidades a través del concepto de variable estable, aquella que no es premonitoria de una transformación y que mantiene su perfil en un período temporal determinado, a diferencia de la variable inestable, la cual constituye el germen del cambio. Pero lo interesante de destacar es que bien se trate de procesos variables o de cambio se mantiene la misma dirección analítica, cuyo punto de partida son unidades nítidamente segmentables, que corresponden a los paradigmas estructurales tradicionales. En consecuencia, también las modificaciones están destinadas a ser observadas científicamente respecto de tales parcelas, como si sólo fuera posible abordar el cambio atomísticamente y no, de manera más amplia y en relación con transformaciones combinatorias complejas como las que atañen a la sintaxis $y, s i$ acaso, a las estructuras discursivas.

De otra parte, Labov subraya el hecho de que los hablantes no buscan intencionalmente la deformación o la transformación de las leyes que rigen el orden de su código linguístico, y por lo tanto las mutaciones tienen lugar a pesar de los hablantes, como consecuencias involuntarias, no buscadas ni premeditadas, de sus propias acciones. Más aún, el autor se refiere a la interpretación idealizada de los fenomenos de la lengua y al rechazo de toda variación desde la perspectiva de los hablantes, de acuerdo con el llamado "principio de la edad dorada» (The golden age principle), que se describe del modo siguiente: «At some time in the past, language was in state of perfection» (pág. 514). Tal concepción de la perfección de la lengua en el pasado se conecta con el supuesto de que todo cambio es interpretado por los hablantes como una forma de corrupción. A partir de aquí éstos realizarían actos contrarios a su voluntad, la cual se encamina más bien hacia la preservación de las unidades de la lengua, y en este sentido siguen una dirección inversa a la variación. Si esto es así, ¿por qué se da el cambio, y por qué justamente, en relación con el aspecto material y más perceptible de la lengua? Pero éstas son preguntas que el autor no intenta responder aquí. 


\section{LOS FACTORES SOCIALES DEL CAMBIO}

En las primeras investigaciones, los factores sociales enumerados y analizados por Labov eran de dos tipos: los referidos a ciertas condiciones propias de los hablantes, sean naturales o adquiridas, sean permanentes o provisionales, a saber: clase social, sexo, generación, etnia, raza; y los referidos a ciertas condiciones derivadas de las situaciones comunicativas y de los textos producidos: los estilos, definidos básicamente a través del grado de la formalidad de la situación, el cual a su vez se mide respecto del control que ejerce el hablante sobre el propio discurso.

Labov insiste, con razón, en la relación contingente e impredecible entre los aspectos de la estructura linguística que serán afectados por la estructura social. Es un hecho que sólo ciertos rasgos linguísticos son objeto de «motivación social» (social motivated projection), y que no es posible determinar previamente cuáles son las características de tales rasgos. En lo que sigue, comentaré los factores que desempeñan un papel central en el fenómeno de cambio.

En la medida en que tanto el sexo cuanto la generación están determinados de modo natural, la clase social es respecto de ellos el concepto más discutido y discutible, por su doble naturaleza, por un lado, subjetiva y, por otro, relativa a las sociedades particulares. Sin embargo, pese a tales consideraciones, no existe modo de sortear la importancia de los valores jerárquicos al estudiar la estructura de cada sociedad. Creo que la cuestión conflictiva se salva si un estudio es lo suficientemente explícito respecto de los valores que definen el índice de clase sobre el que se basará el análisis, y respecto de la justificación que ha llevado a la elección de tales valores en la comunidad analizada. Sin duda Labov cumple tales condiciones, de modo que su unidad de medida no puede aplicarse de modo automático a todas las demás sociedades existentes. Con todo, el estudioso muestra clara conciencia de las críticas del concepto de clase, relacionadas en general con la subjetividad, con la rigidez taxonómica y con el particularismo (v. págs. 58-59). Si bien no intenta en modo alguno dar respuestas claras a todas las críticas; opta finalmente por combinar dos índices complejos ya utilizados en los estudios sobre la sociedad estadounidense, a saber, una medida basada en indicadores objetivos como la ocupación, propuesta por Wamer, con otra basada en indicadores subjetivos, como el prestigio asignado a ciertos grupos, propuesta por Duncan ${ }^{5}$. Ambos índices han sido adapta-

${ }^{5}$ Cf. Lloyd W. Warner, Social Class in America: A Manual of Procedure for the Measurement of Social Status, New York, Harper, 1960; y Otis D. Duncan. «A socioeconomic index for all occupations", en A. Reiss (ed.), Occupations and Social Status, New York, The Free Press, 1961, págs. 109-138. 
dos a su propia investigación por Nakao y Treas ${ }^{6}$. Conforme con tal adaptación, Labov refina el primitivo índice socioeconómico tridimensional basado en la educación, ocupación e ingreso, que había utilizado en sus primeros trabajos, sustituyendo el ingreso por el «valor de la casa» (residence value). $\mathrm{La}$ combinación de seis valores jerárquicos dentro de cada indicador permite un acercamiento más fino a la complejidad de la diferenciación social. Así, por ejemplo, la ocupación, que en la mayoría de las sociedades parece desempeñar un papel protagónico en las diferencias, incluye en el extremo inferior a los desempleados, pasando por los obreros no calificados y los calificados, los pequeños comerciantes, los propietarios o gerentes, hasta el extremo superior en el que incluye a los profesionales y directores de grandes firmas. Por otro lado, los rangos asignados a la educación van desde el nivel inferior de la escuela primaria hasta el superior de los profesionales, pasando por grados intermedios como el high school y el college, y diferenciando además el nivel que incluye la obtención de un grado del que no lo incluye (v. pág. 61). Por último el valor de la casa, va desde 0 a 4,900 dólares, en el nivel inferior, hasta más de 25,000 , en el superior ${ }^{7}$. Resulta obvio que tales índices se adscriben a una sociedad específica, y no pueden aplicarse sin más en cualquier contexto.

Pero a esta clásica lista de factores Labov añade dos más: «mantenimiento de la casa» (house upkeep) y «movilidad social» (social mobility) (cf. pág. 63). El primero contempla las innovaciones y arreglos posteriores tanto del interior como del exterior de la casa que la aleja de su modelo original. Puede tratarse lo mismo de cambios y mejoramientos de la fachada, como de ampliación y redistribución de habitaciones, hasta renovación de materiales y de pintura. El segundo, uno de los más significativos para la caracterización de una sociedad, se presenta como una jerarquía de tres valores, la cual introduce mayor complejidad y dinamismo a las escalas sociales, restándoles la rigidez que las caracterizaba e incorporando la posibilidad de ascenso social a lo largo de la vida del individuo. La movilidad social ofrece, pues, la clave para comprender el funcionamiento de las sociedades modemas en constante evolución a partir de los cambios demográficos ocasionados por los flujos migratorios. De hecho, más que el criterio de clase, el de movilidad social será el verdaderamente caracterizador de los protagonistas de la difusión de los cambios. El índice de movilidad se aplica al grupo familiar y se obtiene a través de una comparación entre la ocupación del jefe de la familia y la de sus padres. La escala incluye: la movilidad ascendente (upward mobility), la estable (stable) y la descendente

\footnotetext{
${ }^{6}$ Cf. Keiko Nakau y Judith Treas, The 1989 Socioeconomic Index of Occupations: Construction form the 1989 Occupational Prestige Scores. GSS Methodological Report $\mathrm{N}^{\circ} 74$, Chicago, National Opinion Research Council, 1992.

${ }^{7}$ Los valores intermedios son: $5,000-9,900 ; 10,000-14,900 ; 15,000-19,900 ; 20,000-24,900$.
} 
(downward mobility) (cf. pág. 66), y en ella los individuos clasificados se sitúan en el punto superior, medio o inferior.

\section{LAS REDES SOCIALES}

Es casi lugar común en las críticas al pensamiento laboviano la afirmación de que éste no toma en cuenta las redes sociales y, en cambio, pone como centro el criterio rígido y estático de clase. Pero Labov recoge las críticas de sociolinguiistas como Milroy de modo constructivo, y revisa el peso y el estatuto de la noción de redes sociales, sin dejar de aceptar el papel fundamental que éstas desempeñan en un análisis fino de las relaciones que se establecen entre hablantes ${ }^{8}$. En efecto, la tipología de tales redes (en su relativa multiplicidad y densidad) guarda una estrecha relación con la movilidad social del individuo y, por lo tanto, desempeña un papel decisivo en los procesos de extensión del cambio. Por lo demás, es natural que los fenómenos de variación y de cambio se transmitan a través de las interacciones comunicativas entre los hablantes, pues éstos no ocupan compartimientos estancos como el concepto de clase social parecería suponer, y más bien establecen diversos contactos que van más allá de los miembros del mismo grupo. Aquí la información sobre los individuos con los que un hablante interactúa más frecuentemente en los distintos dominios (familiar, laboral, amical) y el tipo de vínculo que tal interacción presupone (weak ties frente a strong ties), pueden contribuir a interpretar la dirección innovadora o conservadora de la variación y del cambio. Sin embargo, Labov insiste en la función solo complementaria que desempeña la noción de red social respecto de la de clase: dado que no son conceptos excluyentes, no es posible - y muestro mi acuerdo en este punto- reemplazar una unidad de medida por otra, y aún más emprender un análisis de la variación social apoyándose de modo exclusivo en la información sobre las redes de una comunidad, por lo demás difícil de obtener, sin que el investigador posea como base de referencia la información sobre los índices convencionales de clase (aunque sean simplistas o subjetivos) que clasifican los grupos en gran escala. La red social no viene de ningún modo desestimada como instrumento interpretativo adicional, sino que exige complementarse con otras informaciones. Haciéndome eco del pensamiento de Labov, que en este punto comparto, no me parece

${ }^{8}$ Cf. fundamentalmente Lesley Milroy, Language and social networks, Oxford, Blackwell, 1980; Lesley Milroy y James Milroy, «Linguistic change, social network and speaker innovation», Journal of Linguistics, 21, 1985, págs. 339-384; de los mismos autores: «Social networks and social class: toward an integrated sociolinguistic model», Language in Society 21, 1992, págs. 1-26. 
viable analizar la telaraña compleja de relaciones interindividuales de cada hablante sin un conocimiento previo de la estructura social de la comunidad y de la tipología de individuos en la sociedad bajo análisis, su rango ocupacional y la situación social derivada de él, además de otras características fundamentales a partir de las cuales sea posible dar sentido al propio concepto de red social.

Al incorporar como instrumento analítico suplementario el concepto de red social, Labov se vale de una unidad de medida precisa que denomina «índice de comunicación» (communication index), la cual se adscribe a cada individuo investigado con el propósito de identificar su posibilidad de interacción con los demás, tanto en su entorno local, cuanto fuera de él. Pero es un hecho que el tipo de interacciones verbales entre los hablantes constituye un reflejo de un orden social, como lo afirma el autor: «The term communication index reflects the focus on verbal interaction as the product of social relations» (cf. pág. 335). Se obtienen así cinco valores cuyo propósito primordial es medir la densidad de la interacción del individuo con otros en la cuadra, en el barrio en general y fuera de él. Este punto es especialmente significativo para determinar la posibilidad de extensión de la variación y el cambio desde una localidad pequeña a otra más grande, a través del tipo de interacción de ciertos individuos con otros fuera del alcance restrictivo del barrio. Tales individuos comparten ciertas características que los convierten en innovadores, a saber, se sitúan en el centro de redes sociales que trascienden su entorno inmediato: «Leaders of linguistic change are centrally located in social networks which are expanded beyond their inmediate locality» (pág. 364).

La medida de la densidad se obtiene a través del desarrollo de un cuestionario con preguntas como las siguientes: ¿A cuántas personas de la calle donde vives saludarías, o a cuántas y a cuáles invitarías a tomar un café, llamarías por teléfono, les pedirías un consejo?, y otras similares, para determinar el tipo de relaciones que contrae el individuo dentro y fuera de su área de residencia. Las respuestas se ordenan respecto de valores cuantitativos agrupados en torno a ciertas unidades de medida (por ejemplo, para la pregunta acerca del número de personas de la cuadra con la que se establece cierto tipo de relaciones, se ha elaborado un conjunto de cinco posibilidades, que va desde el rango superior: todos en la cuadra, y los inmediatamente inferiores: 6-10 personas, 3-5 personas, 1-2 personas y ninguna). Pero la medida fundamental se centra en la mención de parte del informante de las personas con las cuales realiza ciertas actividades (como tomar un café, salir fuera, pedir un consejo etc.), o con las que mantiene relaciones estrechas de amistad ${ }^{9}$. Coherente con una actitud científica

9 Este método había sido ya aplicado por una de sus colaboradoras en la realización de este tipo de entrevistas. Véase, Arvilla Payne, The acquisition of the phonological system of a second 
ligada a la observación detallista y sistemática cuya confiabilidad se evalúa a partir de un criterio definido de mensurabilidad, Labov también en este caso convierte los valores cualitativos en valores numéricos (cf. págs. $335 \mathrm{ss}$.). Queda así codificado el número de veces que el individuo en cuestión menciona a alguien o es mencionado por otros, con el propósito de determinar el grado de influencia que éste ejerce sobre los demás. La codificación numérica —que proporciona un efecto de seguridad y precisión cuando se aplica a un asunto tan complejo como el de las relaciones sociales, que escapa a los criterios de organización - también corre el peligro de reducir y empobrecer la información cualitativa acerca de la naturaleza misma de tales relaciones, información que de ser tomada en cuenta prescindiendo del principio de mensurabilidad, permitiría determinar con mayor certeza la calidad y el peso de la interacción entre los individuos y los grupos.

Es interesante destacar, en lo que a las medidas de estratificación social concierne, que el autor las determina sobre la base de un serio estudio de las localidades investigadas, incluyendo un análisis de su composición interna: la historia social, la formación demográfica, y el estatuto que tienen dentro de la ciudad. En verdad, sólo un estudio sociolingüístico mediocre y poco sólido -y el de Labov no puede en modo alguno recibir semejante calificación- podría aplicar una medida ciega de clase social sin haber estudiado primero la estructura de la comunidad por investigar y haber adaptado los instrumentos necesarios para identificar los grupos sociales de modo relevante y significativo. En este sentido, cualquier crítica a Labov al respecto se revela inconsistente.

\section{EL PLANO INDIVIDUAL}

Lo más novedoso de la propuesta de Principles está —a mi juicio- en la búsqueda de una compensación de los análisis reductivos y simplificadores procesados mediante la cuantificación a través de un desplazamiento del foco de atención hacia el individuo, pues solo así puede emprenderse el análisis de las redes sociales. Aquí entra de modo natural el plano estilístico, el cual comporta la consideración de las diferencias situacionales a las que se ve expuesto un individuo en su quehacer cotidiano, y desempeña a la vez un papel fundamental en el origen, y en la transmisión y difusión del cambio. Un estudio ejemplar en esta dirección constituye el análisis de un día de la vida de una

dialect, University of Pennsylvania Dissertation, 1976, y de la misma autora, «Factors controlling the acquisition of the Philadelphia dialect by out-of-state children», en: William Labov (ed.), Locating Language in Time and Space, New York, Academic Press, 1980, págs. 143-178. Labov comenta esta investigación en la página 430. 
informante (Carol Meyers), a través del cual se logra registrar un espectro amplio de variación estilística individual según los diferentes contextos en los que se inserta durante la jornada: familiar (con el marido en la casa), laboral (relaciones con jefes, colegas y clientes en una agencia de viajes de la cual la informante es administradora), y amical (en las sesiones de esparcimiento, como el juego de bridge con un grupo de amigas) ${ }^{10}$. Como resultado de esa observación se encuentra una sorprendente coincidencia de la variación estilística con el espectro de la variación social: mientras más formal la situación comunicativa, las variables utilizadas se acercan más a los prototipos estándares. En cambio, conforme se alcanza un grado mayor de informalidad en las situaciones lúdicas con congéneres, aparecen variantes más alejadas de tales prototipos y más bien correspondientes a grupos de menor jerarquía social. Esta incursión en la escala de la estratificación social a través de las modificaciones en la dimensión situacional de un solo individuo en relación con otros constituye un hito fundamental para interpretar el cambio lingüístico como un hecho que supone un cierto manejo interpretativo de la variación pragmática de la lengua, la cual parece surgir — según Labov- en los primeros años de la infancia, mucho antes de las percepciones que revelan una conciencia de las diferencias de clase social.

\section{LOS PROTAGONISTAS DEL CAMBIO}

El autor se hace la pregunta clave y más importante del volumen, la que supone un verdadero vuelco de la dirección analítica que hasta ahora había seguido: ¿Quiénes son los protagonistas del cambio? (Who are the leaders of change?). Esta se convierte en la cuestión medular de la investigación, la cual supone, a mi modo de ver, una modificación significativa de las versiones anteriores de la teoría de la variación, ligadas a la observación de los factores grupales uniformes y de los índices cuantitativos resultantes, sin singularizaciones entre los hablantes.

Para responder a esta pregunta, el autor analiza la tipología social que subyace a los individuos que han obtenido un alto índice de comunicación, esto es, redes sociales densas en el interior de su localidad y extensas con lazos débiles, y por lo tanto menos densas, con miembros de otros grupos. Labov se detiene de modo pormenorizado en la historia individual de las integrantes centrales y más influyentes del barrio, como Celeste y Teresa. $Y$ termina identi-

${ }^{10}$ Las consideraciones de Labov se basan en el análisis realizado por Donald Hindle, The social and structural conditioning of phonetic variation, University of Pennsylvania, PH.D. dissertation, 1980. Véase el comentario de Labov, págs. 438-445. 
ficándolas como innovadoras en la sociedad analizada, vale decir, capaces de transmitir un fenómeno propio de un grupo a otro. En líneas generales, las innovadoras pertenecen al género femenino, ocupan el centro de la jerarquía social (upper/ower working class), y han obtenido un puntaje alto de movilidad social ascendente (upward mobility). Pero el cambio no se genera por la pertenencia a esos grupos sociales particulares, antes bien su verdadero desencadenante responde a ciertas características individuales que revelan actitudes de no conformismo social (non-conformity). Para evitar malentendidos, hay que subrayar las precisiones del autor en varias partes del texto, en el sentido de que los factores de clase social o de sexo no son per se los verdaderos motores del cambio. Tampoco se trata de descender al plano meramente idiolectal -como podría desprenderse de los análisis del comportamiento individual - para buscar allí las causas de las modificaciones. Los individuos se comportan como innovadores (leaders), no porque sean los representantes de una clase social situada en el centro de la jerarquía, como la de los obreros calificados, ni porque pertenezcan al género femenino. Son innovadores en la medida en que exteriorizan un tipo definido de conducta social que reacciona ante los valores vigentes de una comunidad. En la sociedad norteamericana, según Labov, no son las polaridades de clase o de género las que actúan en la sociedad, sino polaridades más abstractas que revelan comportamientos reactivos ante ciertos valores dominantes, como las de «conformismo» frente a «no conformismo» (conformity / non conformity). Y más todavía: esta oposición tiene mayores alcances que la estratificación grupal. De hecho --según afirma Labov- se trata de la primera oposición que perciben los niños en su proceso adquisitivo de la lengua, la cual se vincula con la variación estilística, ejemplificada en el comportamiento lingüístico de Carol Meyers, comentado líneas arriba. Abordaremos el aspecto cognoscitivo en la siguiente sección.

\section{El PLANo cognoscitivo}

Según lo sostiene el autor, el proceso cognoscitivo de la lengua materna se cumple a través de la percepción de parte del niño de valores estilísticos opuestos, como los expresados en la dicotomía: formal / informal. Así, los aprendices asociarían, por un lado, el polo formal a contextos donde prima la adecuación a las normas o la imposición de ellas, i.e., los ambientes escolares o, en general, aquellos de carácter disciplinario. Por otro lado, el polo opuesto, de la informalidad, terminaría asignándose a los contextos en que se relaja el comportamiento y, por lo tanto, los valores impuestos dejan de tener vigencia en los ambientes más espontáneos, como los lúdicos, entre compañeros de es- 
tudio. Una situación análoga se encuentra en el experimento de la variación situacional de la jornada de Carol Meyers, en la cual resulta patente la acomodación y modificación de ciertos rasgos del habla de la informante respecto de sus interlocutores y de las situaciones comunicativas con las que se confronta. Así, las formas innovadores aparecen en las situaciones relajadas del juego de bridge compartidas con otras mujeres, y tales situaciones constituyen los ambientes favorables al cambio, que ocurre sin que los protagonistas sean conscientes del mismo (change from below).

En su profundización acerca de los mecanismos que mueven a la sociedad estadounidense, Labov toma como modelo de referencia el estudio de Eckert acerca de los patrones que guían la conducta de los adolescentes en un colegio de un suburbio de Detroit («Belten High» en Neartown)". La autora descubre una polarización de pautas definidas que se manifiestan en dos tipos de conducta frente al orden establecido: sumisas frente a disidentes, representadas por el grupo de Jocks y el de Burnouts, respectivamente. Así, son los Jocks los que respetan las nonmas de comportamiento en la escuela, mientras que los Burnouts las transgreden. Entre ambos extremos surge la figura mediadora de individuos más neutrales, que se mueven con comodidad entre uno y otro grupo sin ligarse de modo absoluto y definitivo a ninguno de ellos (los In-betweens). El estudio de Eckert encuentra las variantes más avanzadas de una variable vocálica entre los Burnouts, sobre todo si son mujeres. Pero en la interpretación laboviana que aplica tales hipótesis a un contexto mayor (el cambio en Filadelfia), son los llamados In-betweens los verdaderamente innovadores en la sociedad, pues al mediar entre usos de uno y otro polo, logran expandir las formas de conducta hacia fuera del grupo originario.

Ahora bien, ¿por qué confiere Labov tanta relevancia a un estudio particular de un grupo de adolescentes de una escuela? La respuesta está en su convicción de que tal polaridad de comportamiento, solo aparentemente ligada a la conducta escolar, no hace sino reflejar polaridades sociales más generales, a saber, conformistas frente a no conformistas, llevadas a las últimas consecuencias durante la adolescencia, pero equilibradas en la actividad social adulta. De hecho en la perspectiva laboviana los canales de difusión del cambio se dan a través de figuras dominantes que captan atención o que se consideran influyentes como modelos imitables, para lo cual se requiere individuos que mantengan buenas relaciones con su grupo originario, pero que a la vez sean apreciados fuera de él, de modo que logren entablar relaciones positivas con integrantes de otros grupos. Precisamente los líderes del cambio en el análisis laboviano

"Cf. Penelope Eckert, Jocks and Burnouts: Social Categories and Identities in the High School. New York, Teachers College Press, 1989; y de la misma autora, Linguistic Variation as Social Practice, Oxford, Blackwell, 1999. 
han sido identificados como ln-betweens en la etapa de la escolaridad (cf. págs. 432-436).

Valdría la pena explorar si estos mismos principios que subyacen a los factores sociales ya mencionados se manifiestan de modo similar en contextos diferentes, y qué modalidades describen. Parece difícil postular la validez de una polaridad abstracta de la naturaleza de la propuesta por Labov para interpretar la motivación central del cambio en Estados Unidos, si aplicamos tal propuesta a sociedades en estado de formación - como por ejemplo las hispanoamericanas- a causa de las continuas migraciones intemas, en las cuales los valores institucionales no están claramente establecidos, ni tampoco los medios de movilidad social. En tales sociedades, donde se dan intrincados procesos de mestizaje sin duda motivados por las modificaciones profundas de la composición demográfica, entra en juego otro tipo de percepción y de hermenéutica de parte tanto de los hablantes en proceso de inserción a la nueva comunidad cuanto de los receptores que deben acomodarse a los nuevos pobladores. En estos contextos, oposiciones como la de originario frente a migrante adquieren mayor relevancia en el imaginario colectivo, como resultado de la confrontación dicotómica, por lo menos en las primeras fases de migración. En todo proceso de cambio demográfico (procesos similares se desarrollan actualmente en las sociedades europeas, sobre todo en los países con migración relativamente reciente), se dan las condiciones para el desarrollo de una percepción contrastiva entre lo propio y lo ajeno a la sociedad en que se convive, de parte tanto de los grupos receptores como de los migrantes ${ }^{12}$. Dicotomías análogas han sido desarrolladas - si bien con distinta formulacion y aplicadas a sociedades criollas- por Le Page y Tabouret-Keller, y consideradas por Labov como no aplicables a la sociedad norteamericana (cf. pág. 505) ${ }^{13}$.

\section{EL ESPACIO: LOS BARRIOS}

En la sociolinguística laboviana, entre la lista de los factores externos, no figura el espacio. Y no es que éste no estuviera omnipresente cada vez que se emprendieran análisis sociolingüísticos, los cuales - por lo demás- no dejan de estar localizados en un punto geográfico, sino que carecía del estatuto ope-

${ }^{12}$ He desarrollado este punto respecto de la migración intema de grupos indigenas hacia la capital en el Perí. Al respecto, véase Sociolingutística del español de Lima, Lima, Pontificia Universidąd Católica del Perú, 1990 y «Variedades lingiísticas en contacto. Propuesta de investigación del español del Perí», Signo y Seña (Buenos Aires) 6, junio 1996, págs. 493-511.

${ }^{13}$ Cf. Robert B. Le Page y Andree Tabouret-Keller, Acts of ldentity: Creole-based Approaches to Language and Ethnicity, Cambridge, Cambridge University Press, 1985. 
rativo-funcional de los demás factores sociales, y por lo tanto - hasta donde se me alcanza- - no formaba parte de las operaciones cuantitativas como una variable independiente del tipo de las consabidas de clase, de sexo o de generación. Y a esta situación contribuye el hecho de que las diferencias espaciales sometidas a examen requerirían un número ingente de hablantes representativos asociados a diversos espacios, de modo que fuese posible obtener un peso cuantitativo análogo a los demás factores como para permitir el contraste. El factor espacial, dominante en la tradición dialectológica, el cual -como es obvio- no era objeto de medición cuantitativa sino que desempeñaba mas bien una función tipificadora, perdía aquí su lugar preferencial. Los espacios terminaban siendo sólo nombres identificatorios para delimitar el campo del estudio sociolingüístico de la modalidad de una lengua determinada, cuyo estatuto no se pone en juego. Esta situación se mantiene básicamente inalterada en la obra bajo examen: no entra el espacio como factor del mismo estatuto que los de clase, sexo, generación, movilidad social o etnicidad. No obstante, a mi modo de ver, la espacialidad o territorialidad adquiere una dimensión de mayor relevancia que la concedida en los trabajos anteriores, porque es abordada a través del análisis de microcosmos territoriales como los barrios (neighborhood) dentro de la gran ciudad, análisis que incluye la composición demográfico-social, la categorización de los pobladores y el estatuto del propio espacio en la sociedad urbana más amplia. Una vez más la dirección de la indagación sociolinguística parte del microanálisis y avanza por inducción a contextos de mayor generalidad y alcance. En efecto la justificación de tal camino inferencial reside en que el barrio, como primer punto de ligazón del poblador con su espacio, podría considerarse también el centro originario del cambio si logra proyectarse hacia otros sectores de la ciudad. Labov desarrolla un aparato metodológico analítico de gran precisión para el conocimiento de la estructura social interna de las localidades pequeñas a través del manejo de corpora amplísimos que combinan distintos tipos de indagación extensiva e intensiva ${ }^{14}$, de mayor o menor acercamiento a los individuos. Así, por un lado, el material se recoge a través de entrevistas en las que el investigador se introduce en el barrio y participa en la interacción de sus miembros como si fuera uno de ellos (site study); y, por otro, se utilizan indagaciones como las telefónicas, que tienen un alcance extensivo mayor aunque sean más alejadas desde la perspectiva de la participación del observador (random study). La calidad del corpus sobre el que se basan las observaciones y la organización estratégica de éste se revela como uno de los aspectos que confiere mayor fiabilidad a las hipótesis labovianas.

14 Para estos conceptos, puede verse Rocío Caravedo, «Dialectología y sociolingüística. Una propuesta integradora», La Torre (Puerto Rico) 7-8, 1998, págs. 75-87. 
Ahora bien, cuando se trata de la localización del punto generador del cambio, el barrio adquiere sin duda una importancia capital. Pero no debe perderse la visión panorámica que permite considerar estas localidades pequeñas como parte de una macroorganización (regiones o países) a la cual remiten. Y el carácter de esta remisión, la identidad de los microespacios y los macroespacios, y la interrelación entre ambos varía según las sociedades específicas. Habría que explorar, por ejemplo, qué papel desempeñan en nuestras sociedades hispánicas ambos tipos de espacio, si están relacionados entre ellos, o si coexisten de modo relativamente independiente. Siguiendo con el ejemplo de las sociedades que han experimentado grandes cambios demográficos por migración interna, es indudable la relevancia de la formación de los microespacios, tanto de los individuos migrantes que buscan reconstruir su mundo original o construir otro distinto en el nuevo contexto, cuanto de los originarios que se desplazan dentro de la misma ciudad en bușca de lugares más alejados respecto de lo que consideran el grupo invasor. Paralelamente, se van formando los espacios abiertos o comunes, donde se encuentran ambos grupos y se crean distintos canales y formas de interacción. Pero, al lado de esto, es obvio que los individuos no viven en cotos cerrados sino que, por lo menos en su imaginario colectivo, se sienten pertenecientes a espacios más amplios que en la mayor parte de casos coinciden con los límites nacionales. La realidad de esta percepción cobra fuerza justamente cuando el individuo migra a otro país y tiene que confrontarse con las diferencias, no sólo de los originarios sino de los migrantes de distintos centros de procedencia. En el imaginario colectivo lo que cuenta no son exclusivamente los microespacios: los espacios referenciales tienen dimensiones más amplias y, en general, coinciden con los estados nacionales. Otros problemas surgen, como es natural, en el caso de las regiones coloniales. ¿Se pueden trazar líneas interpretativas que conecten las características y modos de vida de los microespacios con los macroespacios nacionales de referencia? Una pregunta semejante exige insertar junto con otros elementos relevantes para el estudio de la génesis y la expansión del cambio una categoría espacial que no se restrinja al microanálisis de la variación intema en los barrios.

\section{LA ETNICIDAD Y EL CONTACTO DE LENGUAS}

Otra de las posibles críticas a las tesis labovianas reside en el aparente desinterés en el estudio del contacto de lenguas como factor que puede orientar la variación y el cambio linguísticos. Tales críticas podrían justificarse, por lo menos parcialmente, en el hecho de que el modelo de análisis laboviano está 
preparado sólo para abordar la variación interna de una lengua. En el presente volumen, en el que Labov reconsidera sus planteamientos iniciales en una perspectiva más compleja y dinámica como la del cambio, la cuestión de la influencia del contacto con otras lenguas surge ligada al factor de etnicidad. En efecto, la lengua originaria de un grupo es considerada como un rasgo fundamental en el reconocimiento étnico. Según el autor, la categoría étnica se define como forma de identidad social ligada a los orígenes familiares y, por lo tanto, no adquirida de modo exógeno. En sus términos: «Ethnicity is a social identity that is attributed to people by virtue of their descent, an acquired rather than an achieved characteristic» (pág. 245). Tal categoría podría también involucrar otros aspectos como la raza, la filiación religiosa, pero la evaluación y el peso de cada uno de estos componentes depende de cada comunidad. Así, para tomar el mismo ejemplo de Labov, la diferencia entre protestantes y católicos en Irlanda es considerada de tipo étnico, lo que no ocurre en Estados Unidos. Por otro lado, los hebreos - según el autor-constituyen una categoría étnica que no se define necesariamente por la filiación religiosa, a juzgar por el hecho de que tal identidad no se pone en juego aun cuando el individuo cambie de religión. De las afirmaciones de Labov se desprende que, en la sociedad norteamericana los grupos étnicos se identificarían con los migrantes: italianos, polacos, hispánicos, africanos, asiáticos etc., si bien es ostensible la asimetría de la enumeración de los tipos identificados. Tal tipificación tiene sin duda un carácter relativo, habida cuenta de que la nación estadounidense se ha ido formando como producto de diferentes oleadas migratorias y resulta difícil identificar un tipo de población como originario. Claro está que la noción de migrante es relativa al punto de vista de quienes se consideran pobladores naturales del lugar, no sólo por el hecho de descender de quienes participaron en las primeras oleadas migratorias (pues de hecho, por ejemplo, la migración africana tiene una larga data) sino porque pertenecen a un grupo racial y religioso políticamente dominante en la sociedad (no en vano el llamado WASP, White, Anglo-Saxon, Protestant, mencionado por Labov cuando se refiere a la composición demográfica de los barrios, constituye una categoría referencial [pág. 67]). Hasta donde puedo entender, Labov se referiría a los grupos vistos como migrantes a partir de otros que se consideran originarios por el hecho de corresponder a etapas tempranas de migración. El tiempo migratorio parece ser determinante para la percepción de lo originario y de lo exógeno. Aunque la lengua de base ocupa un papel central en la definición del grupo étnico, no parece constituir un rasgo inequivoco para definirlo (piénsese en los africanos o los asiáticos, que pueden tener distintas lenguas de base). En suma, los elementos que juegan aquí expresan más bien formas subjetivas de reconocimiento externo de ciertas afinidades, en algunos casos raciales, en otros, glotológicas, incluso meramente geográficas (bien nacionales: italiano; bien conti- 
nentales: hispano, africano, asiático) por parte de los grupos que se autoconsideran más antiguos o pioneros en un lugar determinado, y no deben ser juzgados a partir de una unidad de medida objetiva y coherente.

Lo cierto es que Labov trata el factor de etnicidad principalmente como referido a la participación de la base cognoscitiva de la lengua primaria del respectivo grupo étrico en los procesos de cambio del inglés. El siguiente texto sintetiza su posición conclusiva respecto del efecto poco significativo de la influencia de tal factor a través de la relación adstratística entre lenguas (no obstante los hallazgos de sus investigaciones más tempranas [Labov 1972], como la de Martha's Vineyard y la de Nueva York: «Given the fact that the ethnicity of speakers is the trait most closely related to language, it is quite surprising to find very little differentiation of the English of the population by ethnicity" (pág. 247). El efecto de la etnicidad en la adopción de ciertas formas linguísticas no es, pues, según el autor, determinante como lo es la influencia de factores de tipo socioeconómico, generacional o genérico (cf. pág. 259).

Comentemos brevemente el caso de los italianos del barrio de «South Philadelphias, especialmente tratado por Labov. Frente a la mayor incidencia de la vocalización de $/ \mathrm{r} /$ interna post-voć́lica, en una zona como la de Filadelfia caracterizada por la retención de tal entidad fónica, se pregunta Labov si la explicación debe buscarla en la influencia adstratística del italiano. Si así ocurriera -continúa el autor- la presencia de tal rasgo disminuiría conforme fuese decreciendo el uso de la lengua de parte de los individuos más jóvenes, que la abandonan a favor del inglés. Pero los resultados no revelan esta conclusión, pues al parecer (salvando y reinterpretando las posibles erratas que se han deslizado en los cuadros, que impiden una interpretación transparente de las correspondencias) la vocalización no disminuye de modo proporcional al abandono del italiano, sino que registra más bien un incremento ${ }^{15}$. No obstante, al contrastar tales resultados con diferencias de sexo y de clase social, Labov observa que los hombres y la movilidad social baja contribuyen a una mayor vocalización, y que por lo tanto no es el factor étnico el responsable del proceso. Queda en pie, sin embargo, explicar por qué precisamente los italianos registrarían este uso diferenciándose de las tendencias de los demás, si no estuviera involucrado de alguna manera el factor llamado étnico. Independientemente de las conclusiones, creo que hay que tener en cuenta que el hecho de que un hablante no use la lengua originaria de sus antepasados no impide que un rasgo compatible con ella pueda transferirse por otras vías a su modalidad inglesa. Los fenómenos implicados en la influencia adstratística no se trasladan de una

15 Tales resultados están expresados lamentablemente de modo un tanto confuso, pues, con toda probabilidad debido a erratas, las conclusiones no concuerdan con los resultados de los cuadros, y no se puede inferir la lógica del razonamiento. 
lengua a otra exclusivamente en el habla de quienes conocen y dominan ambas lenguas, sino que también pueden aparecer aun cuando se desconozca la lengua fuente, mediante un traslado indirecto a través de los diversos y complejos procesos comunicativos entre los hablantes. En tales procesos, los que ya no hablan la lengua de los antepasados pueden recibir la forma en cuestión a través del contacto con otros sujetos de la misma procedencia, que sin dejar de hablar su lengua originaria hablan también el inglés y han trasladado dicha forma a su habla inglesa. Un desconocimiento de la lengua de los antepasados no lleva, pues, necesariamente al abandono de la forma adquirida por parte del hablante, pues ésta ya se ha incorporado a su nueva lengua.

Por lo demás, quizá pueda valer como elemento de juicio para la interpretación de este fenómeno particular el hecho de que la $r$ asibilada del siciliano, estigmatizada en italiano, tiene como rasgo característico la aparición de un elemento sibilante, el cual ya supone un debilitamiento respecto de la forma oclusiva o un desprendimiento del contacto entre al ápice de la lengua y los alvéolos. Si a las características articulatorias se une la estigmatización del fenómeno, no resulta extraña la producción de un sonido intermedio vocalizado, en el intento de evitar el ruido sibilante evaluado de modo negativo. Esta valoración se transmitiría al inglés hablado por los migrantes que poseen el italiano como lengua matema y se continuaría de modo mecánico en los descendientes que han dejado ya de hablar esa lengua.

Sin embargo, existen otros casos que, según Labov, son inexplicables desde el punto de vista linguístico, pero que parecen tener correlaciones con el factor de etnicidad y el efecto adstratístico, a saber, la mezcla entre $/ 0 /$ y $/ \mathrm{oh} /$ entre polacos ${ }^{16}$, el uso de later con el valor opuesto de 'temprano' entre los portorriqueños y la confusión de let y make de parte de los italianos de South Philadelphia. Me detendré brevemente en el último caso. Labov presenta como curiosos los siguientes ejemplos tomados del habla de los migrantes italianos: How could you make your husband dance with all those women?, It would let Jesus cry ${ }^{17}$, en que se muestra la confusión entre make y let. A pesar de aceptar el posible efecto étnico, Labov añade: «We do not at present know of any semantic differences in Italian lasciare and fare and English make and let that would account for this ethnic efect» (pág. 257). Pero justamente tal confusión no puede ser sino un reflejo nítido del amplio espacio de variabilidad semántico-discursiva de fare en italiano, que admite más posibilidades que make, y

16 (oh) constituye una de las variables vocálicas estudiadas por Labov a lo largo de los años, y alude a la posteriorización y elevación de /o/ como proceso de cambio vocático de parte de ciertos grupos sociales en Estados Unidos.

17 Las formas estándares de tales enunciados serían: How could you let your husband dance with all those women? ('Como puedes permitir que tu marido baile con todas esas mujeres') y $I t$ would make Jesus cry ('Esto haría llorar a Jesús') [mía la traducción de ambos enunciados]. 
que se usa en muchos casos con el valor del inglés let (fammi parlare= let me talk). Tal uso justifica de modo plausible la confusión del par let/make en el inglés de los migrantes italianos o de sus descendientes. Este sería, pues, un caso de clara influencia adstratística.

Existe un aspecto digno de comentario en el volumen de Labov, y es la interpretación de los grupos hispánicos en relación con la marginalidad de la influencia del factor étnico. Al igual que en relación con el componente africano, Labov afirma que tales grupos no siguen las tendencias generales de cambio del inglés norteamericano y, da por supuesto que no influyen en modo alguno en los procesos evolutivos de esta lengua, en la medida en que se trata de grupos cohesionados entre sí, marginales en el complejo de la sociedad americana. Las modalidades propias de inglés utilizado por esos grupos no transgredirían los límites interétnicos. Habría que averiguar si esa conjetura se cumple en los puntos de mayor concentración de la migración hispánica (la cual por lo demás ha crecido notablemente en los últimos años), sobre todo cuando se producen contactos diversificados entre descendientes de migrantes y originarios.

\section{TRANSMISIÓN, DIFUSIÓN Y CONTINUIDAD DEL CAMBIO}

En los áltimos capítulos, que constituyen el punto culminante del trabajo, Labov distingue tres fases del cambio: «transmision», «difusión» y «continuación" (transmission, incrementation y continuation). La primera de ellas, en la cual se detiene de modo especial, puede enfocarse tanto desde el punto de vista social como individual. En el primer sentido, se centra en la expansión externa de las formas a través de espacios mayores en los cuales las redes sociales y las relaciones comunicativas entre los hablantes desempeñan - como ya hemos visto-- un papel fundamental. Pero el germen individual no sólo de la transmisión sino también de la difusión (incrementation), y por lo tanto de la continuación (continuation), hay que buscarlo - -según el autor- en el proceso mismo de adquisición lingüística, a través del cual el niño desarrolla un sistema cognoscitivo de su lengua en el ambiente familiar. Resulta obvio que el niño está en condiciones de captar tanto el sistema de invariantes de su lengua cuanto el de variables estables, esto es, aquellas que se mantienen en determinado período temporal dentro de ciertos márgenes. Pero un mecanismo meramente imitativo y reproductor de las variables existentes no permitiría explicar el surgimiento del cambio, sino solo el mantenimiento y la uniformidad del sistema. El niño aprende - según esta lógica laboviana - un sistema de variación de carácter estable, cuya permanencia se da a través de la repetición o de la generalización entre los grupos de las condiciones internas del sistema. Volvamos a estas condiciones intrasistemáticas. 
En el primer volumen de Principles, Labov había propuesto la intervención de un mecanismo biológico universal llamado «emparejamiento de la probabilidad» (probability matching), cuya aplicación a la lengua permitiría la reproducción de los diferentes rangos de generalidad de las variantes adscritas a distintas variables de su lengua, y en esta medida la adquisición del sistema de variabilidad de la lengua fijado en ciertas coordenadas espacio-temporales ${ }^{\mathrm{t}}$. Nótese cómo la variación así entendida describe, como ya lo he apuntado antes, el mismo comportamiento que la invariación: permanencia de un estado de cosas, conservación, uniformidad. En este sentido, la variación interna no permite concebir el cambio (si bien Labov muestra lo contrario cuando aborda los factores internos que lo favorecen), y ésta parece corresponder más bien a una perspectiva invariacionista de las lenguas. No son, pues, los llamados factores internos los verdaderos anticipadores del cambio. Para explicar éste, hay que imaginar algún tipo de perturbación del orden dado, del sistema de estabilidad, y esa perturbación no puede venir sino de la intervención de elementos extraños al sistema, los llamados factores externos. Labov enfrenta el problema de la transmisión del cambio dentro del proceso de adquisición lingiuística como «reorganización del vernáculo» (vernacular reorganization), el cual supone la interpretación social de los hechos de tipo lingüístico, y, por lo tanto, la modificación del orden recibido en las etapas tempranas de tal proceso. Ahora bien, tal interpretación, que tiene sin duda un componente individual, no es absolutamente arbitraria ni accidental: Labov intenta encontrar las raíces de tales perturbaciones interpretativas en la primera oposición de opcionalidad que hipotéticamente recibe el niño y que — según su interpretación- no es tanto de carácter social cuanto estilístico. Como ya he comentado anteriornente, la oposición formal/informal es la que entra aquí en juego, y ella termina asociándose a una dicotomía mas bien de tipo ambiental, a saber, disciplinario/no disciplinario, identificada por un lado, con los ambientes institucionales de la escuela o con las correcciones de los adultos, y, por otro, con los ambientes lúdicos cuyos integrantes son los compañeros generacionales.

Pero ¿cómo se realiza la reorganización del vernáculo? Se trata de sucesivas reinterpretaciones de la variación recibida a partir del ambiente familiar, especialmente de la madre o sustitutas de ella en el cuidado del niño (female caretakers). En otras palabras, el punto de partida del cambio tiene como base el sistema comunicado a los niños por las mujeres que están a cargo de ellos. Labov ejemplifica el asunto con varias investigaciones, de las cuales es paradigmática la de la localidad de Milton Keynes realizada por Williams y Kerswill en un barrio de migrantes en las cercanías de Londres (págs. 425-

18 Cf. William Labov, Principios del cambio lingülstico. Vol. I Factores internos (versión española de Pedro Martín Butragueño), cit. nota 1, págs. 882-910. 
427) ${ }^{19}$. Un rasgo innovador de esta ciudad es la glotalización de $/ t /$ Los niños de Milton Keynes adoptan las formas heredadas de sus madres o nodrizas, propias del dialecto londinense, incluso el rasgo aludido, pero van desarrollando simultáneamente en el proceso evolutivo generacional un reacomodo de tales formas hasta llegar a una intermedia, un tanto distinta de la originaria que, sin embargo, puede considerarse como la nueva forma local derivada y reinterpretada en el contexto de los coetáneos. En el análisis cuantitativo, se muestra con claridad que hasta los 4 años, los niños reproducen el sistema de sus madres, y conforme se van acercando a la adolescencia cambian el patrón y se distancian unos grados más de tales formas acercándose a las de los demás jóvenes de su entomo.

La reorganización del vernáculo propuesta por Labov implica una readaptación continua de los individuos que tiene como base las reinterpretaciones de ciertos rasgos del habla de los adultos en la preadolescencia, a partir del legado recibido por las mujeres a cargo de dichos individuos. Los rasgos sujetos a reinterpretación son obviamente aquellos considerados sobresalientes, desviados de la media (outliers), en la percepción de los aprendices.

La difusion (incrementation), que es la fase siguiente a la de transmisión del cambio, alcanza una cima durante estas etapas, después de las cuales puede registrar algunos descensos. Por ello Labov propone un modelo de curva logística de incremento del cambio como más apropiado que uno lineal, haciendo proyecciones estadísticas muy elaboradas de la dirección del cambio en diferentes períodos temporales. $\mathrm{El}$ análisis que parte de la infancia del individuo y del sistema heredado no implica que los adultos no sean protagonistas del cambio, pues justamente aquellos fenómenos que evolucionan son los que no se adscriben de modo exclusivo a una etapa provisoria de cambios generacionales, sino que se mantienen, aun si con menor énfasis, o avanzan gradualmente durante la adultez. Desde sus primeros trabajos Labov estableció una clara diferencia entre las modificaciones provisionales de la etapa adolescente que no continúan en la madurez del individuo y que, por lo tanto, no provocan cambios en la lengua de la comunidad (age-grading), y las modificaciones que sí corresponden a cambios en progreso y que bien pueden continuarse en línea ascendente o estabilizarse en cierta fase alcanzada (generational change). Estas modificaciones que se dan en un «tiempo aparente» (apparent time) requieren la contrastación empírica en el decurso temporal real (real time) . La continuación (continuation) del cambio como fase culminante del proceso está circunscrita a los límites de la percepción de los hablantes, en la que - por lo

19 Cf. Ann Williams y Paul Kerswill, «Dialect levelling: continuity vs. change in Milton Keynes, Reading and Hull», en P. Foulkes y G. Docherty (eds.), Urban Voices, London, Arnold, 1999. 
menos para el inglés según las hipótesis labovianas-- los puntos sobresalientes (outliers) en las secuencias fónicas desempeñan un papel decisivo en la dirección o en la desviación de los procesos. Tales puntos sobresalientes se materializan en los monosíabos acentuados en el espacio lingüístico angloamericano analizado por Labov. Sería interesante plantearse en qué tipo de materialidad lingüística se concentraría la percepción de los hablantes de español con el propósito de identificar los puntos más sensibles al cambio en esta lengua.

\section{LOS PRINCIPIOS DEL CAMBIO LINGÜISTICO URBANO}

Quizás uno de los aspectos más prometedores para la investigación futura de la propuesta laboviana es el modo como se conectan los aspectos sociales del cambio con los cognoscitivos individuales en el proceso de adquisición de una lengua. Tal convergencia queda plasmada en las siguientes fases de los principios del cambio linguístico en las grandes ciudades (Principles of Urban Linguistic change) (pág. 437), postuladas por Labov teniendo como punto de referencia el proceso de reorganización del vernáculo:

1. la herencia recibida de las madres o nodrizas como punto de partida del cambio;

2. reinterpretación de parte del niño de las diferencias existentes en términos de la polaridad informal /formal;

3. adscripción de tal diferencia a la polaridad social, respecto de la cual una forma tolerada en la situación informal sería posteriormente calificada como correspondiente a un grupo social inferior ${ }^{20}$;

4. asociación de la situación informal con el no-conformismo a las normas (change from below) en los jóvenes;

5. adopción de parte de cierto tipo de adultos de las formas no conformistas.

El autor no deja de reconocer que tales fases corresponden al contexto de las grandes ciudades, y que son otras oposiciones reinterpretativas las que parecen regir el cambio en ambientes rurales.

Labov subraya además que los principios que rigen el cambio no parten necesariamente de un grupo referencial determinado (de género, de clase social, de grupo generacional o étnico), aunque los resultados de su investigación

${ }^{20}$ Esto es bastante relativo, puesto que también as frecuente que las formas de registros informales sean consideradas como propias de un grupo social superior o prestigioso que se erige como un modelo de imitación para los demás hablantes. 
indiquen a un solo género (femenino) perteneciente a una clase social determinada de la jerarquía (la clase central: lower/upper working class), como protagonista del cambio. En efecto, tal estado de cosas constituye sólo el resultado de circunstancias sociales particulares. La verdadera causa del cambio responde -según el autor - a una lógica distinta cuyo punto de partida es la percepción de valores generales en relación con conductas conformistas y no conformistas de la sociedad. Estas tendencias quedan formuladas en dos principios aparentemente contrarios. El primer principio se define del modo siguiente: «1. The Non conformity Principle: Ongoing linguistic changes are emblematic of nonconformity to established social norms of appropriate behavior, and are generated in the social milieu that most consistently defies those norms» (pág. 516).

Según este principio llamado de «no conformismo», los procesos de cambio revelan actitudes no conformistas de los individuos en relación con las normas sociales establecidas, y se originan en ambientes sociales en los que tales normas se transgreden de modo consistenté. Pero no basta que las personas sean exponentes del no conformismo social, como se deduce el primer principio, sino que es también necesario que ellas constituyan modelos de referencia imitables para los demás, de acuerdo con lo que el autor denomina un «principio no conformista constructivista» (constructivist non conformist principle). Textualmente: $\ll 2$. The Constructive Nonconformity Principle: Linguistic changes are generalized to the wider community by those who display the symbols of nonconformity in a larger pattern of upward social mobility".

En otras palabras, los individuos que satisfacen tal requisito son -de acuerdo con este segundo principio- aquellos que cumplen condiciones de representatividad y que no sólo actúan dentro de su área local sino que son capaces de ejercer influencia en grupos ajenos a los de su procedencia originaria. Aquí vuelve a concentrarse el autor —aunque sin explicitarlo- en el criterio de prestigio desarrollado en sus primeros trabajos, si bien tal criterio adquiriría un contenido distinto si lo reinterpretamos respecto de los nuevos principios enunciados aquí. No está de más añadir que la noción de prestigio es ciertamente relativa a las circunstancias histórico-sociales de cada lugar.

FINAL

De la riquísima propuesta de investigación referida y comentada en estas páginas sólo he podido rozar la superficie de algunos problemas que, sin duda, concitarán la atención de la investigación futura. Los estudios que marcan una época en la evolución del pensamiento disciplinario - $-\mathrm{y}$ no cabe duda de que éste lo es- son aquellos que no solamente se limitan a ofrecer soluciones fijas 
a problemas determinados sino que son capaces —sobrepasándose a sí mismos - de proponer nuevas cuestiones, mostrando vacíos pertinentes y planteando dudas significativas o relevantes que encaminen y dirijan la investigación posterior.

Interrogantes como las siguientes exigen un cambio de orientación de la percepción científica. ¿Por qué unos rasgos están sujetos a cambio mientras que otros no? ¿Qué hace que los individuos en las etapas tempranas de la vida aprendan un sistema aparentemente fijo, pero a la misma vez impulsen de modo inconsciente solo algunas modificaciones del mismo, mientras que retraigan otras hacia formas más conservadoras? La respuesta debe venir de un estudio de la dirección perceptiva de los hablantes. Labov ha tocado, si bien por ahora solo lateralmente, este problema cuando se refiere a los rasgos sobresalientes (salient outliers) que recaen en los grupos monosilábicos, esto es, las entidades fonéticas que concentran en una lengua específica como el inglés la atención de los hablantes, y que, por lo tanto, son permeables a las modificaciones. ¿Pero se ejercita - y de qué modo-- la percepción también sobre unidades mayores con contenido semántico (léxicas o morfosintácticas)? De otra parte, ¿son las estructuras o las modalidades discursivas objeto de percepción? ¿Qué papel juegan éstas en los procesos de cambio? ¿Se debe extender el foco de percepción analítica de las unidades mínimas segmentables a los conjuntos combinatorios complejos, como las totalidades del orden discursivo o textual?

Al conectarse con el dominio cognoscitivo, probablemente las cuestiones señaladas estén reservadas al tercer volumen prometido por el autor (Cognitive Factors). Queden, por consiguiente, estas preguntas como pendientes de respuesta.

Mientras tanto, la gran empresa de Labov, plasmada en este libro, nos pone frente a un sinnúmero de desafíos: sólo una investigación empírica minuciosa sobre las cuestiones medulares que he subrayado a lo largo de la presente exposición permitirá validar, matizar, contradecir - si acaso solo reforzar o enriquecer- las conjeturas formuladas, haciendo posible su extensión a otros contextos sociales, como el de las sociedades hispánicas, pendientes de estudio riguroso y teóricamente unitario. 